\title{
Design, Upgrading and Creation of the State-of-the-Art Two- Photon Laser Microscope View of Features Used in Neuroscience
}

\author{
Alexander Popov ${ }^{1,2+}$ and Maxim Doronin ${ }^{1}$ \\ ${ }^{1}$ Lobachevsky State University of Nizhny Novgorod, Nizhny Novgorod, 603950, Russia \\ ${ }^{2}$ Volga State University of Water Transport, Nizhny Novgorod, 603950, Russia
}

\begin{abstract}
This article may be regarded as a quick reference to laboratory staff who wish to develop their own microscope system for self- service and modernization in order to save the lab budget. Two-photon microscopy offers several advantages compared with other forms of microscopy. Of particular importance is two-photon microscope for biology, since it allows research on living tissue with less damage to it, while having a higher resolution, the ability to penetrate to a greater depth and has less dissipative. The use of a pair of photo multipliers allows to use two dyes with various ranges of fluorescence that is important for performing biological researches. Detectors are installed in purpose designed place to the aperture of lenses, besides, the fluorescence beam doesn't pass the way back through the scanning module, but directly gets onto the detector. They are accommodated on the movable part of the microscope. The work is performed with financial support of the state represented by the Ministry of Education and Science of the Russian Federation, the unique identifier of the project is RFMEFI57814X0079 project, the agreement on granting a subsidy 14.578.21.0079 dated 28.11.2014 between the Ministry of Education and Science of the Russian Federation and UNN.
\end{abstract}

Keywords: two-photon microscope, imaging, uncaging, programming, engineering, designing.

\section{Introduction}

Today one of the main areas of application of multiphoton microscopy is biology. This is due to the fact that this technique allows to obtain 3D images of tissues due to laser focus change (1), that is possible due to substantially greater penetration depth on the main wavelength into biological tissues (2). However, there are certain peculiarities arising from the specifics of experiments using this technique. In one case, for example, the analysis of the morphological cell data of hippocampal slices of the brain of rodents, other experiments in vivo depending on the design using the full-sphere of the virtual reality (3) or, for example, in the form of a cylindrical hoverplatform (4). Specific features are also in the fact that the level of fluorescence-activated objects is low (only a few suites), and the increase of the laser intensity may overheat and burn the sample (5). Sample scan methods are constantly changing, and require constant modernization. Creation of twophoton system taking into account its specific use in neuroscience is probably the best solution.

\section{Main Types of Microscopes}

Devices of different designs are used to observe fluorescent objects. A device in which the fluorescence excitation of the object is performed by laser and the fluorescence image of the object is recorded with a CCD camera. The laser is placed to the side of the sample that is illuminated at an angle. The image of the sample is created using two identical photographic lenses arranged to meet each other and transmitting images of the object 1: 1 . One of the drawbacks of the system is its insufficient sensitivity that requires a longer (about 10 minutes) exposure time for image recording. Two-photon microscopy offers several

\footnotetext{
+ Corresponding author. Tel.: +79524707259

E-mail address: popov@neuro.nnov.ru
} 
advantages compared with other forms of microscopy. Of particular importance is two-photon microscope for biology, since it allows research on living tissue with less damage to it, while having a higher resolution (290 nm lateral, axial $500 \mathrm{~nm}$ ), the ability to penetrate to a greater depth $(0.6-1 \mathrm{~mm})$ and has less dissipative (Svoboda and Yasuda 2006). With this method, conducted studies on dendritic spines (diameter 0.5-1 mm) and astrocytic processes of (Ding 2012; Grienberger and Konnerth 2012; Stosiek et al. 2003). However, the disadvantage of the two-photon imaging is the high level of noise, and moreover, there is a diffraction limit. These two factors limit the resolution of the system (Cole, Jinadasa, and Brown 2011). Therefore, in addition to improvements in the optical elements of two-photon microscopes mathematical methods are often used to increase the resolution of systems and improve the signal / noise ratio. An example of such a filter is Gause filter and newly developed Block-Matching 3D (BM3D) filter (Danielyan et al. 2014). BM3D filter has a better signal / noise ratio performance and significantly reduces the cost of optical elements to increase the resolution of the two-photon images.

For carrying out research with the method commercial models of two-photon microscopes were developed. Such devices are present at a model range of the companies that are engaged in production of optical microscopes and accessories for them, for example, Nikon (A1RMP), Olympus (FV1000MPE), Carl Zeiss (LSM810 NLO). However these microscopes have an unsatisfactory indicator of detecting of low level fluorescence, for example, at research of calcium emission into extracellular space on neurons and astrocyte of the brain arising at activation of postsynaptic receptors (Clapham 2007). This problem can't be solved by increasing the intensity of radiation as it can lead to burning out of the sample. Changing of scanning methods is also impracticable task as the factory guarantee doesn't envisage intervention of the end-user in the device. Besides, the service centers of the producers presented work with a delay (as a rule, some months, during this time a scanning method can change again), and services of the centers demand serious financial expenses. Thus, it is necessary to develop a new design for detecting low level fluorescence with the possibility of performing experiments of various classes and the possibility of fast change of methods.

\section{Designing of the Microscope}

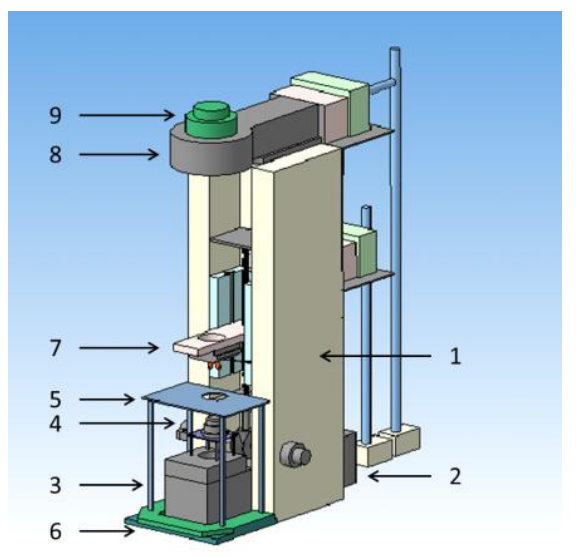

Fig. 1: 3D model of Microscope

The model depicts the main frame (Fig. 1,1), attached to the anti-vibration table, as well as optical elements, attached to the main frame. Technically, the operation of DIC is provided with installation of a visible light source (Fig. 1, 2) and an IR filter, an iris-shutter, a diffuser, a mirror to reflect the beam in the vertical direction and lens (Fig. 1,3). After passing through the lower block and being reflected the light beam reaches the polarizer, and then passes onto a condenser (Fig. 1, 4) with Nomarski prism. Then a ray of light hits the sample, which is fixed on the table (Fig. 1, 5). The table, in turn, is fixed to a U-shaped moving platform (Fig. 1, 6) for exact positioning of the sample under the lens in the lateral plane. Passing through the sample, the beam hits the optical components on a moving platform (fixed to the frame of the microscope) (Fig. 1, 7), and passes the second Nomarski prism and the analyzer. Here the interference of previously separated rays is performed. After passing the mentioned optical path the light beam falls onto the so-called illuminator of reflected light (Fig. 1, 8), that does not contain any filters or mirrors in the DIC mode 
operation, and then the beam passes onto IR camera (Fig. 1,9) that displays the image on the monitor.

To improve the quality of the resulting images it is necessary to use not only good program support but also to employ the advantages of the technical (physical) characteristics of the microscope, on the one hand, helping to move the lens to the sample as quickly and often as possible, and on the other hand, providing the most accurate movement when scanning a sample. The design must take into account all these features. In the first case (rapid movement to the sample) it is advisable to use a mechanical movement using a larger number of gear, and in the second case - the minimal number of the gear and electronic movement.

The electric drive control system for practicing a given angle or motion uses feedback sensors providing data concerning the angle or position of the output shaft of the operating engine. If the actuator motor employs a synchronous stepping motor, it is possible to dispense with the feedback sensor (DR) and to simplify the engine control system (CS), as there is no need to use a digital-to-analog (DAC) and analog-todigital (ADC) converters in it.

In addition, in this series there is semi-step and microstep mode when SHM tension is supplied onto windings of the engine forcing the rotor to stop-rise between steps in an equilibrium state which is supported by different levels of voltage-tension on the windings. These significantly improves the accuracy, speed and noiselessness of operation, but the moment decreases and the complexity of the control program increases greatly because it is necessary to calculate the voltage for each step. In our case the control will be exercised by means of the remote control.

The next step is to switch the microscopy system into two-photon mode. The fundamental chart of twophoton microscope is presented in Fig. 2.

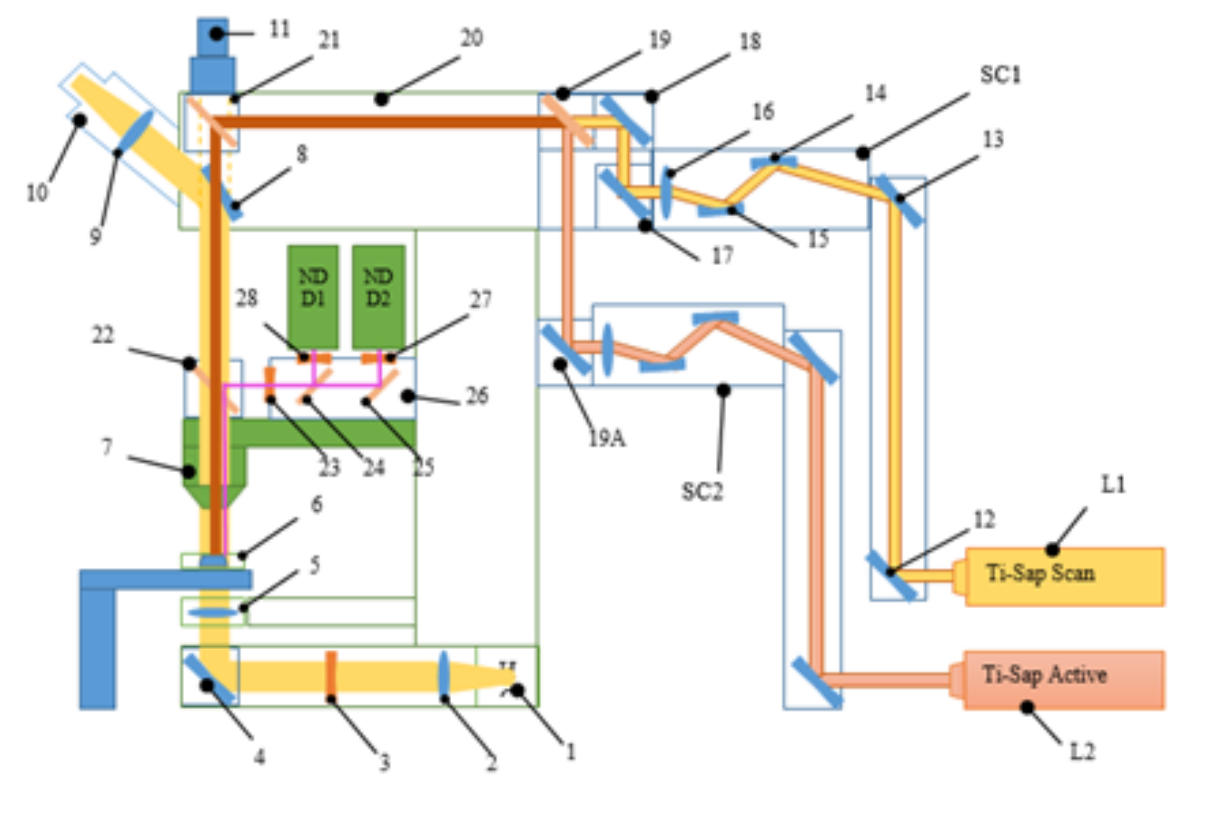

Fig. 2: Optical path scheme

The optical way from the scanning laser to the sample is described below. There is femtosecond laser (Fig. 2, L1) with the frequency of impulse repetition of $100 \mathrm{MHz}$ and duration of $140 \mathrm{phs}$ as a source. The laser allows to adjust the wavelength automatically in the range from 690 to 1080 nanometers. Average output power of radiation makes 1.6 W. Starting its way from the laser (Fig. 2, L1) and passing the optical way including the optical-acoustic modulator (AOM) for changing the radiation intensity and the Galilei telescope for altering the diameter of the beam bunch, the beam gets into the vertical optical bar where there is a mirror (Fig. 2, 12), being reflected from which it is brought to the next mirror (Fig. 2, 13) that provides the delivery of the beam to the necessary height.

Then the beam gets into the resonant scanning block which by means of synchronous operation of the mirror and resonant galvanometers (Fig. 2, 14, 15) and the focusing lens (Fig. 2, 16) scans the sample. 
Parameters of scanner operation are controlled by the computer that allows to exercise high-quality control. By means of mirrors (Fig. 2, 17, 18) the beam gets onto the combining element, it is a dichroic mirror (Fig. 2, 19) which lets the beam getting from the scanning laser pass through and reflects the beam from the activating laser. Then the beam is focused in one plane in the element (Fig. 2, 20) in which, being reflected from a mirror (Fig. 2, 21), passes through a dichroic mirror (Fig. 2, 22), being focused in the necessary plane by means of optics (Fig. 2, 7), and gets onto the sample (Fig. 2, 6), activating its fluorescence in the necessary point, and, point after point (on a trajectory which by means of the computer is set to performance by the CK1 element), scans the whole sample. Photons of light are taken by the lens aperture (Fig. 2, 7), reflected by a dichroic mirror (Fig. 2, 22), pass the filters (Fig. 2, 23, 27, 28) and get on detectors (NDD).The main feature is that the distance from the sample to the light-detectors is 8-9 centimeters, that allows to register even the low level of fluorescence. It is necessary to provide the availability of several protective filters, as during the process of registration of low level fluorescence of the sample there is a high probability of capturing "undesirable" light, for example, laser photons. Therefore the beam passes through the first filter, letting through the wavelength of $400-680 \mathrm{~nm}$, then the beam reflected from the splitter (Fig. 2, 24), having passed through the filter (Fig. 2, 28) $(500-560 \mathrm{~nm}$ ), gets onto the detector (Fig. 2, NDD1), and the passing beam is filtered (Fig. 2, NDD2). The optical path of the laser beam from the activating laser (Fig. 2, L2) is similar to the path from the scanning laser (Fig. 1, L1), but in this case a couple of mirror galvanometers is used as the scanning element (Fig. 2, SK2). The level of fluorescence is processed by the computer. The analysis of the data obtained is carried out by the program MatLab complex. The use of a pair of detectors (photo multipliers) allows to use two dyes with various ranges of fluorescence that is important for performing biological researches. To perform it we use femtosecond pulse IR laser with adjustable wavelength, the optical-acoustic modulator and the element operating it, the scanning modules and elements operating them, detectors (photo multipliers) and elements operating them, the central control system in the form of the personal computer with the program of management of process with operating elements connected to it.

In the process of using a femtosecond pulsed IR laser with adjustable wavelength, acousto-optic modulator and manage element scanning modules and manage the elements, detectors (photomultiplier tubes) and manage the elements, the central control system in the form of a personal computer with a working control program and is connected to it to manage different parts elements. It is also necessary to configure an optical path which is different from the optical path in the DIC. You also need to turn off household light sources and, where possible, to close the microscope does not transmit light box or cloth.

The next step is to switch the system mode of the two-photon microscopy. In the process of using a femtosecond pulsed IR laser with adjustable wavelength, acousto-optic modulator and manage element scanning modules and manage the elements, detectors (photomultiplier tubes) and manage the elements, the central control system in the form of a personal computer with the program management process since it is connected to the various control elements It is also necessary to adjust the optical path which differs from the optical path in the DIC. Besides, it is necessary to switch off household light sources, and also it is desirable to cover the microscope with a box or fabric to shield it from the light.

After preparatory procedures the main operation of two-photon microscopy begins. After searching the area of interest and the switch over to the two-photon mode, the researcher uses a central control system. First, the researcher gets a sample image and adjusts the focus of the lens for scanning in the required plane. Next, the researcher sets the scanning parameters: scan mode, the size and details (in pixels) of the image (if the scan mode is a rectangular area), the scanning speed. Scan mode may include scanning of a rectangular (including a square) area, along a line or a point. More than that, if there are two scanners one can select a scan mode for each of the scanning units.

It should be noted that resonant scanner due to the principles of its operation can only be used to scan a rectangular area. Galvanometric scanner, by managing the position of the laser beam at every moment (in the lateral plane), can be used for various scanning modes, scanning a rectangular area, scanning along a line or scanning a point, as well as photoactivation (uncaging) in particular, glutamate (7). Besides before scanning the researcher can select the wavelength of the laser beam in the central control system. In modern lasers the wavelength tuning process is automated and takes little time (the rate of change of the wavelength is about 
$40 \mathrm{~nm} / \mathrm{s})$.

Detectors are installed in close proximity to the aperture of lenses, besides, the fluorescence beam doesn't pass the way back through the scanning module, but directly gets onto the detector. They are accommodated on the movable part of the microscope. The use of several dyes simultaneously is often the necessary requirement in multiphoton microscopy. Therefore, the design of the microscope detector allows to make fast change of emission filters and dichroic mirror. The standard cube for filters mounted on the slider like "swalow-tail" that is easily inserted and taken out from the detector is used for this purpose and significantly facilitates the work of the researcher. The multiphoton detector must also be equipped with means of electronic protection and a shutter preventing the damage of the photo multiplier and increase in its performance term.

\section{Conclusion}

As a result the creation of the own two-photon system taking into account all special characteristics allows to lower the expenses and to increase the quality of methods helping to improve the quality of the obtained data, enables to conduct researches on live samples with the minimum damage of fabric, makes possible to carry out researches of the data of morphological slices of mice hippocampus, as well as researches on live samples, that is carrying out experiments in vivo. Due to the optimal location of photomultipliers the fluorescence from the activated objects is caught optimally, that allows qualitatively to visualize the object, and does not result in an overheat and burning down of the sample.

\section{Acknowledgements}

The work is performed with financial support of the state represented by the Ministry of Education and Science of the Russian Federation within the Federal target program "Development of Optical Methods of Research of Functioning of a Brain in Norm and Pathology", the unique identifier of the project is RFMEFI57814X0079 project, the agreement on granting a subsidy of 14.578.21.0079 dated 28.11.2014 between the Ministry of Education and Science of the Russian Federation and UNN.

\section{References}

[1] Christopher D. Harvey, Forrest Collman, Daniel A. Dombeck, and David W. Tank 2009 Nature, Vol.461,Issue 7266

[2] W.Denk,J.H.Strickler, and W.W.Webb 1990 Science, Vol.248,Issue 4951, P. 73-76

[3] B.J.Tromberg, N.Shah, R.Banning and others 2000 Neoplasia. Vol.2 Issue2

[4] Mikhail Kislin, Ekaterina Mugantseva' others and Leonard Khiroug 2014 Journal of Visualization Experiments. Vol.88

[5] L Song, E J Hennink, I T Young, and H J Tanke 1995. Biophys Journal Vol.68,Issue 6, P. 2588-2600

[6] David E. Clapham 2007. Cell Vol.131,Issue 6, 2007, P.1047-1058

[7] Diana L Pettit, Samuel S.-H Wang, Kyle R Gee, George J Augustine 1997. Neuron. Volume 19, Issue 3, P.465471

[8] Svoboda, Karel, and Ryohei Yasuda 2006 Neuron 50: 823-39 